\title{
Framework of Construction Procedure Manuals for PMIS Implementation
}

\author{
Boong Yeol Ryoo \\ Department of Construction Science, Texas A\&M University, College Station, USA. \\ Email: bryoo@tamu.edu
}

Received April 20 $0^{\text {th }}, 2013$; revised May 22 ${ }^{\text {nd }}, 2013$; accepted May $30^{\text {th }}, 2013$

Copyright (C) 2013 Boong Yeol Ryoo. This is an open access article distributed under the Creative Commons Attribution License, which permits unrestricted use, distribution, and reproduction in any medium, provided the original work is properly cited.

\begin{abstract}
Primarily project procedure manuals are intended to enforce company polices or procedures. These manuals are important pieces for successful project management in the construction industry, because construction projects are operated virtually. Even though subcontractors are responsible for field works, a general contractor is still responsible for providing quality project management services for project owners. The more subcontractors involved, the harder it is to monitor and control them, due to the different management processes and procedures they use. More than 36 procedure manuals in the building and industrial construction were reviewed to create process maps of various management services. According to surveys, larger contractors have a broader use of procedure manuals than smaller contractors. The full-scale manuals cover project administration, schedule, cost, contract administration, quality, or safety to home office operations. The small-scale manuals cover from site mobilization, to startup and closeout. Small-scale operation manuals are often used by medium to small contractors. This paper presents suggestions for integrated procedure manuals for construction management firms based on studies of multiple construction procedure manuals. A framework for integrating procedure manuals is presented. The proposed framework can be used to keep uniformity across management procedures and phases. In addition, it can be used to implement a project management system. It can be used to forecast or evaluate management activities through replicability of the management responses.
\end{abstract}

Keywords: Procedure Manuals; Action Words; Project Management System

\section{Introduction}

\subsection{Background}

As part of their response to a request for proposal (RFP), a general contractor submits a set of project management manuals to the owner as an element of the contract documents. These are used to determine the level of management competitiveness of the general contractor. The manuals define necessary management tasks, processes, and procedures with included forms for those procedures. It is an essential piece for successful project management, because, while the management team and the subcontractors are connected through the project, each of themhas different management control.

In construction, only a small number of general contractors actively implement project management procedures to describe how they manage building construction projects. The majority tend to only use the documents and forms in the manual to track project issues, manage project budgets, and document issues for legal reasons.
For those who do actively use these procedures they are given an advantage. Because most of them are in a specialized limited number of construction sectors, it helps them to develop a management plan, examine past performance or improve management performance and methods if they have a written procedure manual [1].

The specialization of construction companies and a lack of standardization in construction project management hinder effective communication and documentation between contractors. The main issue in a relationship between contractors is neither of the parties wants to give up their management procedures, because both parties have built their entire systems on these procedures and there is difficulty in changing over to another system.

The end result of this lack of standardization and minority of companies that actively manage with procedures is that there are insufficient procedures for management tasks and commitments. This often leaves managers no choice but to rely on their past experience and individual knowledge to deal with managerial challenges. 
In the short run this usually proves to be sufficient but this non-replicable style makes it difficult to study project performance to determine effective procedures. Also, a non-replicable style makes it difficult to have predictability for results, or incidents in a project.

While there are several professional groups in the construction industry there is still a lack of standards that could be applied in this arena. For example the Construction Management Association of America (CMAA) has released a list of standard services of construction management (CM) in seven management areas [2]. This is representative of the nature of the industry, constructors know what needs to be delivered; they just have a difference of opinion on how to deliver the final product. Because of this, neither specific processes nor procedures are suggested. This lack of specificity leaves it to the individual general contractors to be responsible for creating instructions for construction managers. This responsibility sometimes falls to the individual construction manager, who is given a set of general rules and deliverables and is left to come up with their own method of delivering within those rules.

To aid in construction management, project management information systems (PMIS) have been adopted as a project controlling and monitoring tool. PMISs enable project managers to collect, distribute, measure, and process project data. Initially, large contractors had a tendency to use a custom designed PMIS for their management systems. Recently, however, they have started to adopt an off-the-shelf PMIS and adjusting their management systems to the PMIS. Mainly this has been done due to the expense associated with a customized software system as opposed to one that is mass produced.

Because the majority of the companies use non replicable styles, procedures have become unique to not only each company but to each manager in that company. Thus, synchronizing an off-the-shelf PMIS and procedure manuals are a challenge because this method of operation [3].

\subsection{Purpose of the Study}

The purpose of this paper is to propose a framework for integrated project management in the building industry. By studying processes and methods of existing procedure manuals, a base structure for project control and monitoring procedures is proposed.

Guaranteed Maximum Price CM (GMPCM) projects are an intended target because their management responsibility includes both project management and construction operations. This gives them an opportunity to manage, perform and report on the projects they are involved in. Another feature of this framework is a list of management tasks mapped to illustrate various types of ma- nagement needed at the different phases of a construction project. This would serve as a planning tool for a GMPCM to help in determining which of their management personnel need to be involved in the project, and at what time. The end result of that would be to give them more control of how the projects are to be managed.

CMAA's action words were studied to define the level of management responsibility and describe procedures in a management sequence. While this list does not supply specific processes, it does provide predefined words whose universal meaning makes it possible to create an understandable framework. These words also were used to determine if instructions within the framework contradict each other.

The proposed framework can be used to optimize the use of a PMIS when there is a disagreement between what is offered by the PMIS and procedure methodologies. It can also be used to restructure existing project procedure manuals to reconcile with the PMIS. This framework would provide a useable form of standardization to help with both this restructuring of existing procedure manuals and aid in the creation of new procedure manuals.

\section{Current Use of Procedure Manuals}

\subsection{Preliminary Studies}

A preliminary survey was conducted if contractors have enough written procedure manuals to effectively execute a construction project. Procedure manuals from Agency CM (ACM), Guaranteed Maximum Price CM (GMPCM), and owner-CM (OCM) contractors were mainly studied to see if there are significant differences among their management areas. The procedure manuals were categorized by management area for direct comparison. Another study showed large contractors' procedure manuals cover a wide range of management tasks from project administration, schedule, cost, contract administration, quality/safety to home office operations. In contrast, medium or small contractors' manuals focus on selected tasks such as site mobilization, startup, and closeout.

As part of the study of procedure manuals of contractors of various sizes the differences between a PMIS and the general contractor's procedures were studied. This study shows that PMISs were limited to collecting and distributing data, in other words a journal and accounting tool for a project. Procedure manuals were used to instruct project managers how management tasks should be executed. The trend usually though is that these manuals are used to supply the deliverable for each procedure.

\subsection{Scope of Standard CM Services}

Construction management is a professional management 
service which applies to construction projects through controlling scope, time, cost, contract, and quality [4]. While there are some smaller builders who perform all of the work involved in a construction project the majority of time the main method involves the use of sub-contractors. Subcontracting is a primary delivery method to access an outside resource to perform a certain amount of work in the construction industry. The cost, quality and speed that come with working with subcontractors bring a few challenges. As each of these subcontractors is their own company they have their own reporting methods, document controls, and accounting. Without a well-organized management guideline, it is difficult for the constructor to effectively work with subcontractors due to the different management types of the subcontractor and the constructor.

CMAA's service map focuses on professional management practices in seven management areas such as project, cost, time, quality, contract administration, safety management, and program management. Nearly 200 tasks are defined from pre-design phase, design, procurement, construction, and post-construction phase [2]. Each management area contains a series of construction management tasks in five project delivery phases. A definition and level of responsibility for each task are provided.

General contractors have been implementing their own $\mathrm{CM}$ procedure manuals at the project level long before CMAA published the standard CM services in 1986. The CMAA's Construction Manager Certification Institute (CMCI), which administers the Certified Construction Manager program. This program is accredited by the American National Standards Institute and this standard was developed as part of CM certification. The standard contracts were developed to achieve the owner's goals [2], and serve as a way for owners and builders to communicate what the end result of a construction project will be.

It becomes an operational issue whether no integrated management system and procedures are available to implement or multiple systems and procedures are available. If there are none then there is the issue of having to create a new system and the costs and delays associated with that. With multiple systems and procedures there is the issue of interoperability between the systems and conflicts of the procedures, while there is no readily measurable costs there are the costs caused by delays, errors and the time spent in an effort to coordinate the systems. A revision of the systems is necessary to choose required services to meet the $\mathrm{CM}$ service requirements based on the size of the project organization structure, operation policies, owner's requirements, and project monitoring and control methods. Care should be taken to ensure that the proper system is chosen.

\subsection{Organization of Procedure Manuals}

Most CM procedure manuals focus on management processes and procedures in the construction phase, from project organization to project closeout. Figure 1 shows a typical organization of the $\mathrm{CM}$ procedure manual. Large contractors tend to own a range of procedure manuals from site mobilization to project closeout. Normally the manuals are edited for each project. Based on the scope of project management, tasks, associated procedures, and particular forms are selected.

A task consists of at least three parts: one part explains what the intended purpose of the section is and why the procedure is important; another to assign the primary individual to implement the procedure; and the other to describe the steps to carry out the procedure. Often forms to fill out are provided to report the results of the procedure. Typically a set of manuals is edited based on the $\mathrm{CM}$ service requirements for the project before the construction phase. The procedure manuals are used more to support operational issues than professional management practices.

The participants include general contractors working in the U.S. and abroad: seven out of top 50 general contractors and four of them ranked in the top 50 contractors working abroad. According to the procedure manuals, about 330 reports are implemented by a general contractor on site. These reports are to cover all areas of the construction project, from finance/accounting, to business development, to industrial relations, to constriction support. Most of them $(69 \%)$ are used to directly support construction operations such as controlling time, cost, or procurement. Quality control and reporting to assist field managers are also a focus. In addition, subcontract monitoring is one of the important components in construction support. 17 percent of the forms are related to

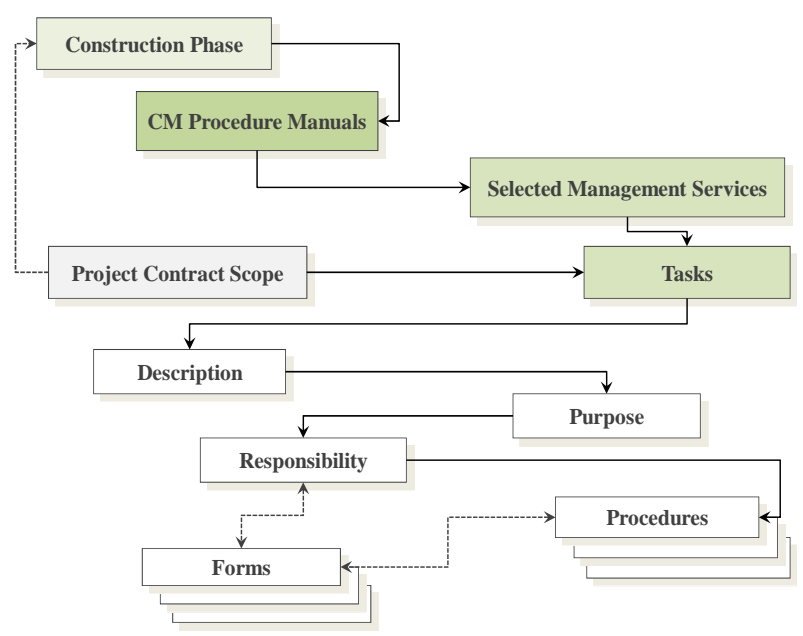

Figure 1. Structure of CM procedure manuals for construction phase. 
finance/accounting and general accounting. The purpose of the forms is to monitor budget and expenses including payroll during the project period. Business development (3.6\%) focuses on preconstruction support. Industry relations $(10.6 \%)$ cover employment, public relations, and safety issues.

\subsection{Use of Procedure Manuals}

According to the survey, most procedure manuals are for site project operations. The scope of CM services is selected according to the management requirements of the request for proposal (RFP). In comparison the agreements created by the CMAA's describe the management areas and major tasks for two types of CM project delivery methods: Agency CM and GMPCM.

More than 36 procedures from 17 construction management $(\mathrm{CM})$ companies were studied and the procedures are focused on site operation. Agency CM (ACM, $6 \%$ ), guaranteed maximum price $\mathrm{CM}$ (GMPCM, 65\%), and owner-CM (OCM, 29\%) companies were included.

OCM procedures are larger than GMPCM's and ACM's procedures are typically the smallest. One of the OCM procedures includes nearly 40 management areas including typical owner tasks such as financing, engineering management, or owner supply items. GMPCM procedures cover 16 to 20 areas of project control at the corporate and site levels [5]. ACM procedures are the smallest in term of service areas and cover management services of contractually requested. Thus it is hard to describe the character of ACM procedures.

Table 1 shows the service modules described by the selected contractors. Of note, larger contractors tend to have an integrated procedure to provide seamless construction management services. These included project administration, time, cost, contract administration, quality, and safety. The three cost related service areas such as scope, time, cost, and contract management are crucial since they focus on controlling and monitoring of schedule/cost. These contractors have the resources to create these seamless systems, and also tend to focus on schedule and cost controls. Larger contractors also had extensive document control procedures as part of their manuals.

For the medium and small contractors, site operation was a primary concern. Their manuals tend to have sectional procedures of specific tasks such as pre-construction, mobilization, project closeout, public relations, or commissioning. A schedule/cost module was also included as a core module. However, procurement or contracting was included but was not fully integrated with the schedule/cost module. Base modules such as project administration policy or project numbering systems were used by larger contractors.
Table 1. Service areas covered by selected procedures.

\begin{tabular}{|c|c|c|c|c|}
\hline \multirow{2}{*}{ Service Areas } & \multicolumn{4}{|c|}{ Contractors } \\
\hline & A & B & $\mathrm{C}$ & $\mathrm{D}$ \\
\hline Management \& Administration & $\sqrt{ }$ & $\sqrt{ }$ & $\sqrt{ }$ & $\sqrt{ }$ \\
\hline Organization \& Responsibility & $\sqrt{ }$ & $\sqrt{ }$ & $\sqrt{ }$ & $\sqrt{ }$ \\
\hline Project Control & $\sqrt{ }$ & $\sqrt{ }$ & $\sqrt{ }$ & $\sqrt{ }$ \\
\hline Scope Management & $\sqrt{ }$ & $\sqrt{ }$ & $\sqrt{ }$ & \\
\hline Time Management & $\sqrt{ }$ & $\sqrt{ }$ & $\sqrt{ }$ & \\
\hline Cost/Estimating & $\sqrt{ }$ & $\sqrt{ }$ & $\sqrt{ }$ & $\sqrt{ }$ \\
\hline Safety Management & $\sqrt{ }$ & $\sqrt{ }$ & & \\
\hline Quality Control/Inspection & $\sqrt{ }$ & $\sqrt{ }$ & $\sqrt{ }$ & \\
\hline Contract Administration/Procurement & $\sqrt{ }$ & $\sqrt{ }$ & $\sqrt{ }$ & $\sqrt{ }$ \\
\hline Project Numbering System & $\sqrt{ }$ & $\sqrt{ }$ & & \\
\hline \multicolumn{5}{|l|}{ Project Management System } \\
\hline Document Control System & $\sqrt{ }$ & $\sqrt{ }$ & & $\sqrt{ }$ \\
\hline Progress Reports & $\sqrt{ }$ & & $\sqrt{ }$ & $\sqrt{ }$ \\
\hline Change Management & $\sqrt{ }$ & & $\sqrt{ }$ & $\sqrt{ }$ \\
\hline Delays \& Claims & $\sqrt{ }$ & & & $\sqrt{ }$ \\
\hline Quality Assurance & & & $\sqrt{ }$ & \\
\hline Quality Plan & & & $\sqrt{ }$ & \\
\hline Request for Information & $\sqrt{ }$ & & & \\
\hline
\end{tabular}

Design management procedures were occasionally found since it is not a required task in building construction projects. Design control, drawings and specifications, design review were only found in industrial or heavy/ civil projects.

Necessary but special services such as constructability review, risk analysis, value engineering, and life-cyclecost analysis were developed as additional procedures since they require design engineering and construction engineering knowledge.

\subsection{Current Practices in Computerization}

A PMIS is an integrated information system originally intended to assist project managers to collect, analyze, or distribute project information [6]. Generally a PMIS contains predefined business processes and procedures, to aid in data collection and dissemination Lee [7] verified that the chance to complete a project successfully can be increased by $75 \%$ if the right PMIS is implemented.

Three approaches are generally used for computerizing the CM business process to create a PMIS: commercial PMIS, the "off the shelf" products (51\%), custom-built 
PMIS, literally created for the needs of the construction company (18\%), and the hybrid PMIS, a commercial system with some custom built modules added on (31\%). While only a portion of the contractors have an entirely custom-built PMIS, in order to keep the consistency of their internal processes and procedures, a significant number of them would prefer a custom-built PMIS.

No evidence suggested these services are fully computerized. Some contractors implemented a custom built PMIS which was developed based on the contractors' procedures. Their forms and procedures are fully embedded in the PMIS. For contractors who use a comercial "off the shelf" PMIS often use their own forms because they do not want to give up their existing management procedures. In essence they have a computerized system while they continue to fill out their own system of papers. Because these forms are not fully embedded in this type of PMIS this makes some contractors hesitant to implement a commercial PMIS. When it is used, the PMIS is often being used as a data collection, analysis or distribution tool. What is not being used is the ability of a PMIS to fill out multiple types of forms with one set of data. Time is being spent adding the same data multiple times into different forms used by the contractor.

Another issue, due to the expense, custom PMISs have frequently not been updated as the procedure changes or a new project management method is introduced. This widens the difference between the procedures and a PMIS. As a result, most contractors rely on off-line management with the PMIS being used more as a data management tool instead of a project management tool. This negates the effectiveness of the PMIS and discourages the implementation of the PMIS at the project level.

\section{Proposed Framework of Procedure Manuals}

To propose a framework which integrates management modules, studies if standard bidding practices, project management and their functions, management tasks. The management areas were divided into two groups: project control modules (scheduling, budgeting, and contracting) and other supporting modules. Action words and management tasks were studied to propose a framework not only for integrated management but also for business process management of PMIS.

\subsection{Proposed Framework}

The proposed framework for the integrated management of time, cost, and contract is illustrated in Figure 2. By defining the scope of a project, schedule data, budget data, and procurement/contracting data can be fully integrated. The framework calculates progress measurement

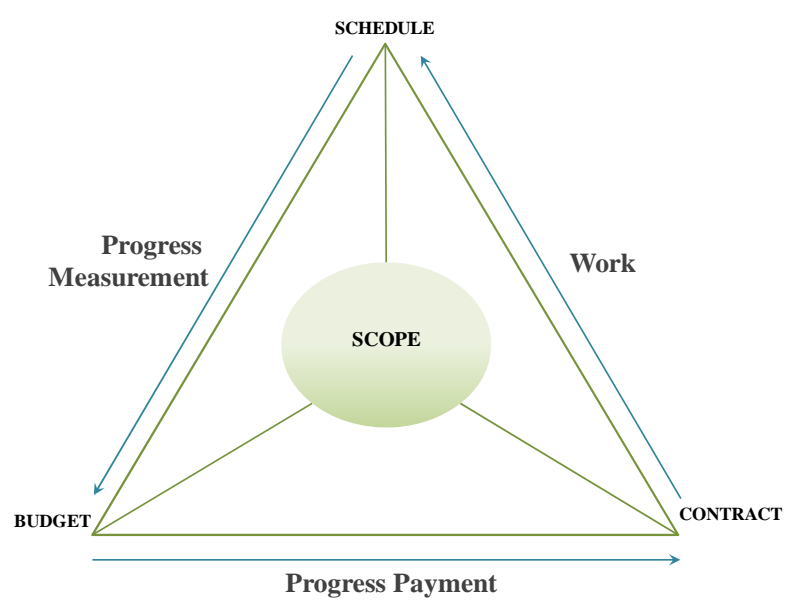

Figure 2. Core management services.

and progress payment easily. This framework also allows managers to update or revised the scope of project when a change order is considered.

Figure 3 maps out how the CM services should support each other at various stages of construction business operations. When the RFP is announced, general contractors submit a bid package in order to be considered for the project. A bid package includes all CM plans which are produced based on procedure manuals with selected management modules. Eventually, work process frameworks can be concentrated into the agreement phase when the general contractor's submittal is reviewed and meets the owner's requirements. Management information can be, simultaneously, collected, organized, and transmitted to the owner using the PMIS.

Figure 4 shows the minimum four levels recommended to combine project management information into one pool of knowledge.

At Level 1, information of the project progress, the project cash flow, and the contract changes is critical. Three pieces of information should be accessed through an activity. Thus not only all deliverables of the project but also the control level activities should be identified. By defining the project deliverables (scope), the remaining management components are connected through the relationships between change orders, progress measurement, and progress payments. When a change order is approved, the scope will be revised and the schedule can be revised. A progress payment can also be made when a progress is measured.

At Level 2, any information affecting scheduling, budget, and contracting should be considered. Creating a project work breakdown structure (PWBS) is critical; the scope of the project is identified. Resource information should be attached so that cost related analysis can be completed. Measuring the time or cost impact caused by a change or delay becomes possible. This also allows 


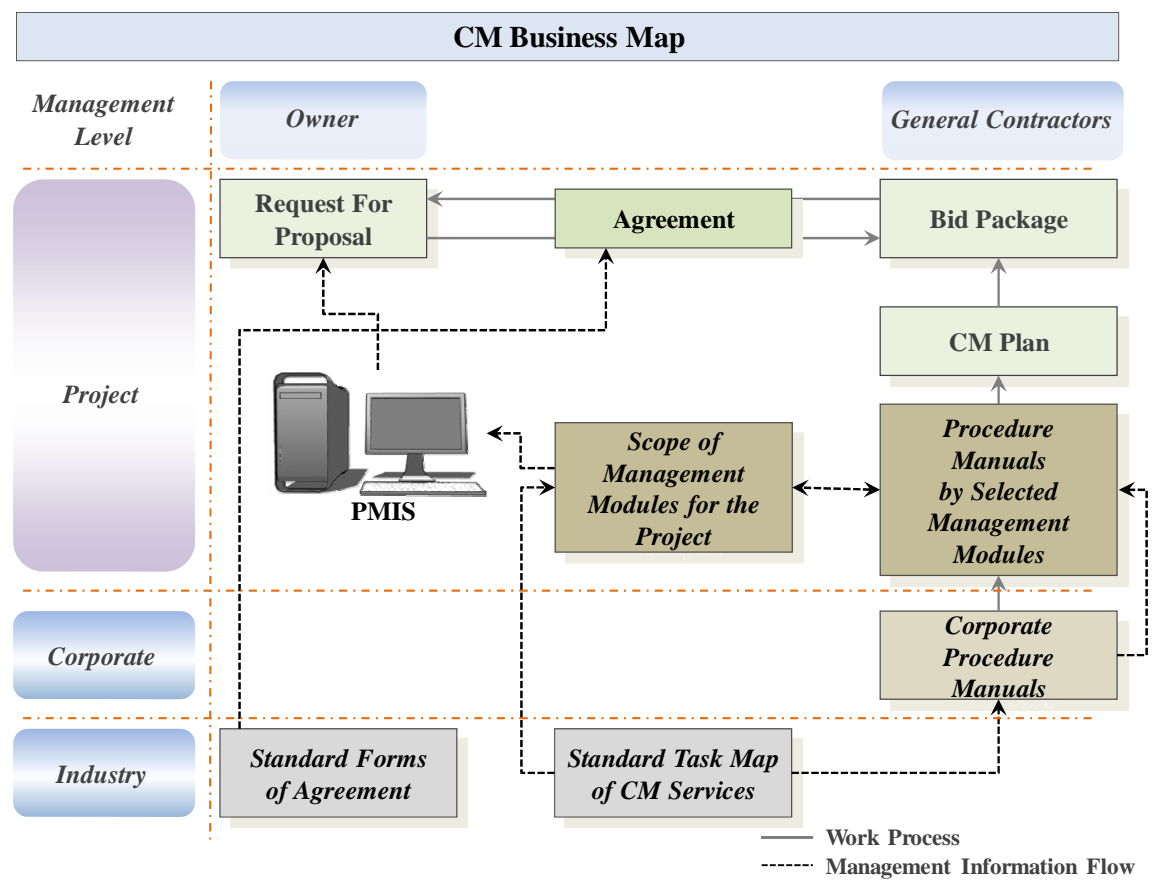

Figure 3. Proposed management framework (Revised from [2]).

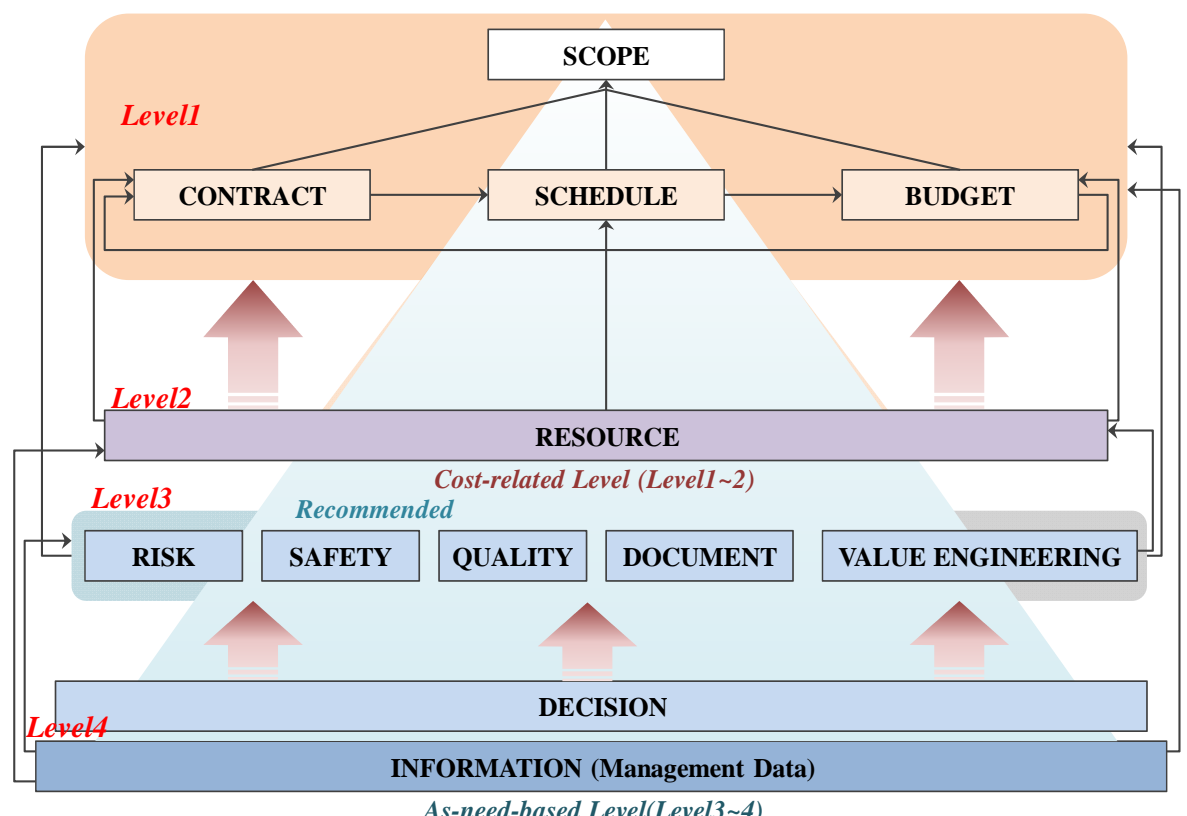

Figure 4. Hierarchy of key management modules.

resource allocation and planning at the corporate level. This can be implemented relatively easily because most large contractors implement the earned value method (EVM) for this purpose.

At Level 3, non-cost information such as safety or quality can be attached. Technical modules such as risk analysis, value engineering can also be included in this level. These modules are supporting modules. Thus they can be developed and integrated independently since they produce information that is needed for limited decision making. Owner requested service, e.g., value engineering or constructability review can be added to this level.

Level 4 contains the decision making module which illustrates how project information is used to make a management decision. Due to a difference in management strategies or operation policies of a contractor, it 
requires a custom framework.

\subsection{Tasks and Procedures Integration Map}

To provide quality $\mathrm{CM}$ services, at least twelve management modules are suggested; budget, project administration, contract, schedule, quality, safety, resource, material/equipment, decision, risk, information management, and value engineering [8]. As Uren [9] suggested ontologies, documents, annotations that link the ontologies to the document, and the users of the systems are implemented. This method was employed to increase reusability and configuration management of the framework [8]. As in Table 2, about 63 action verbs were identified from the CM agreements to define the level of responsibility in construction. These verbs are the core elements of the ontology of the framework.

Figure 5 shows an ontology map for schedule management, monitoring schedule compliance, with proposed tasks and procedures. The map shows action verbs to describe the management needs, management goals, and project data required for the task. In order to remove any ambiguity, each action verb must be defined. Thus the effects of each word can be traced.

A management module consists of a chain of management tasks. A task could include multiple actions. An action word is selected and connected to a management action. A series of management actions are then arranged by chronological management order of each as shown in Figure 5. Management actions can be traced or observed.
Table 2. List of action words.

\begin{tabular}{|c|c|c|c|}
\hline address & develop & investigate & propose \\
\hline administer & discover & issue & provide \\
\hline apprise & discuss & maintain & quantify \\
\hline approve & distribute & make & recommend \\
\hline arrange & document & manage & record \\
\hline assemble & establish & measure & reduce \\
\hline assess & estimate & meet & report \\
\hline assist & evaluate & modify & review \\
\hline assure & expedite & monitor & revise \\
\hline attend & explain & negotiate & require \\
\hline author & facilitate & notify & summarize \\
\hline chair & follow-up & open & tabulate \\
\hline clarify & formulate & organize & transmit \\
\hline compare & forward & pay & update \\
\hline conduct & identify & perform & verify \\
\hline contact & implement & plan & \\
\hline coordinate & incorporate & prepare & \\
\hline define & initiate & price & \\
\hline determine & interview & produce & \\
\hline
\end{tabular}

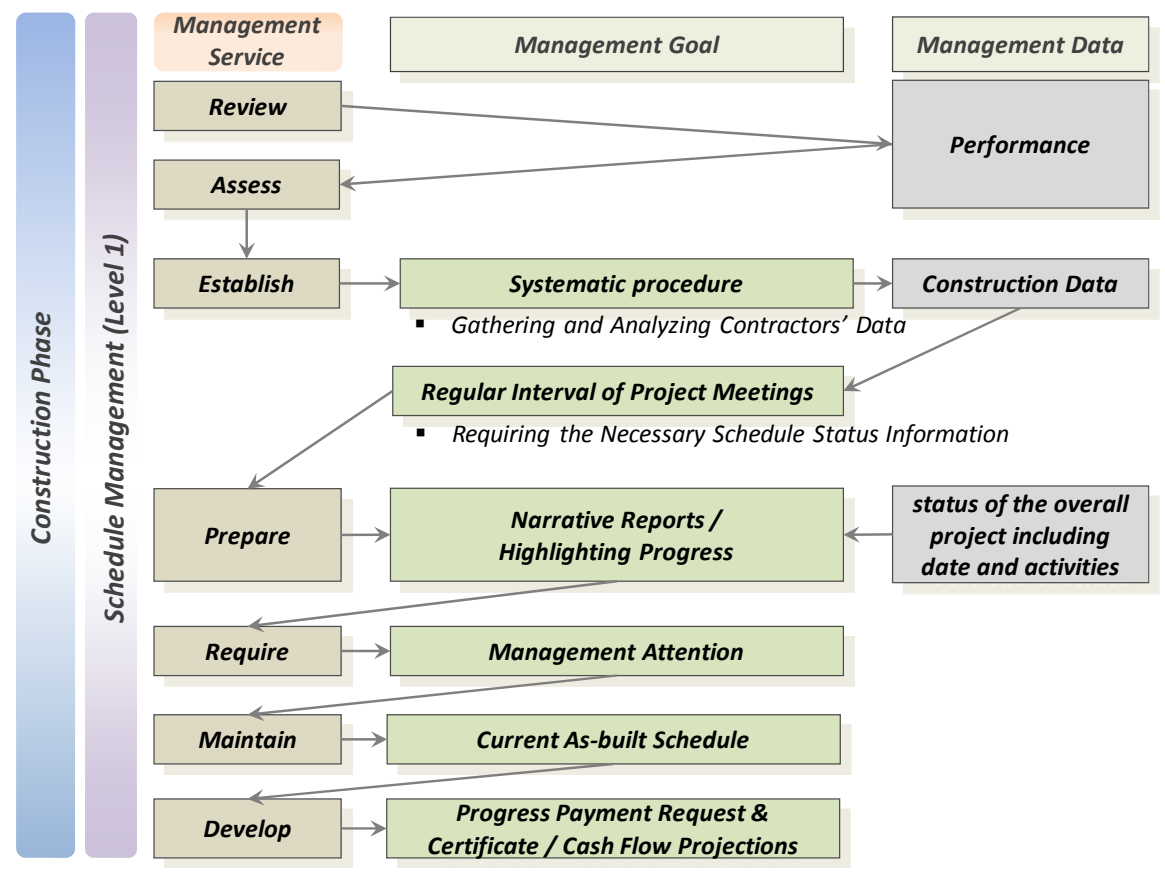

Figure 5. Sample framework of the proposed tasks and procedures integration map. 
It is critical to use the same action words in the procedures and PMIS to remove any misinterpretation. They also explain the level of obligation of each task. A chain of actions words are then used to define the accountability of each responsible party.

\subsection{Suggestions in PMIS Development}

To effectively develop a PMIS, business process modeling is required. It requires a study of current business processes and how the procedures will be reengineered to improve effectiveness. After the reengineering has taken place or if the procedure does not need improvement then the procedures can be used to select a PMIS that fits the management requirements if a commercially available PMIS is considered.

This first part of this procedure is used in the creation of a custom built PMIS. The reengineered procedures are then used by the programmer to create the custom made system to match up with the procedures and reports. For a hybrid system, if the commercial system does not have anything to match up with some of the procedures and the procedures are deemed necessary then customized modules are created to address those procedures.

By following a standard software development methodology, management processes and procedures will be mapped out. Interactions between PMIS and other information systems such as the enterprise resource planning (ERP) system, supply chain management (SCM) are commonly considered. The industry specific software including scheduling and estimating is also considered. This can save time in the development phase since the procedures, especially forms, are what describe document transactions between systems. Thus integration among management systems can be achieved easily and in a manner that makes implementation at the project level more effective due to the system method matching the project level interactions.

\section{Conclusion and Future Work}

Based on studies of more than 36 procedures manuals from construction management companies, the following observations are summarized:

Full service modules are implemented by large contractors whereas medium or small contractors implement a limited number of modules solely for site operations. These are: schedule/cost-oriented, document controloriented, and site operation-oriented.

Large contractors focus on the controlling and monitoring of the entire project. This is to assist construction managers at sites as well as home offices. A schedule/ cost-oriented framework was used to measure the performance of the project using a schedule and cost information. Extensive project operation procedures are often used to cover a wide range of particular $\mathrm{CM}$ services such as VE, LCC, CR, risk analysis, or public relations.

Level of integration of the procedures with comercial PMIS is relatively low. The PMIS is mainly used as a central data repository for project control or document control. Off-line construction operations are still handled by separate procedures. This drops the efficiency of PMIS implementation. This makes contractors hesitant about purchasing a commercial PMIS package. Finding a PMIS package that fits the corporation's project control procedures is a difficult process.

Medium or small contractors tend to implement modules for site operations at the project site such as preconstruction, mobilization, commissioning, and project closeout. Because of a lack of technical analysis, trending and forecasting are challenging. Formulating procedures in the project control areas are necessary and computer software should be employed to cover missing project control methods.

Even though the PMIS is an effective data processing tool in project management the synchronization of offline operations and on-line reporting procedures is recommended in order to improve the efficiency. As part of the procedure manual the functionality requirements of the PMIS should be described. Thus selection of a PMIS with synchronized management modules is important.

\section{REFERENCES}

[1] Y. Lin and H. Lee, "Developing Project Communities of Practice-Based Knowledge Management System in Construction," Automation in Construction, Vol. 22, 2012, pp. 422-432. doi:10.1016/j.autcon.2011.10.004

[2] CMAA, "Construction Management Standards of Practice," Construction Management Association of America, 2010.

[3] C. Thomsen, "Program Management Version 2.0," Construction Management Association of America, 2008.

[4] CMAA, "An Owner's Guide to Construction and Program Management: Enabling Project Success under Any Delivery Method," Construction Management Association of America, 2011.

[5] B. Y. Ryoo and J. Kang, "Impact of CM Procedure Manuals in Construction Project Management," 3rd International Conference on Construction in Developing Countries, Bangkok, 4-6 July 2012.

[6] A. Jaafari, "Towards a Smart Project Management Information System," International Journal of Project Management, Vol. 16, No. 4, 1998, pp. 249-265. doi:10.1016/S0263-7863(97)00037-9

[7] S. Lee and J. Yu, "Success Model of Project Management Information System in Construction," Automation in Construction, Vol. 25, No. 10, 2012, pp. 82-93. doi:10.1016/j.autcon.2012.04.015

[8] C. Haltenhoff, "The CM Contracting System: Fundamentals and Practices," Prentice Hall, Upper Saddle River, 
1998.

[9] V. Uren, "Semantic Annotation for Knowledge Management: Requirements and a Survey of the State of the Art,"
Web Semantics: Science, Services and Agents on the World Wide Web, Vol. 4, No. 1, 2006, pp. 14-28. doi:10.1016/j.websem.2005.10.002 